\title{
Electron Beam Channeling in Single Atomic Column
}

\author{
Anudha Mittal and K. A. Mkhoyan
}

Chemical Engineering and Material Science, University of Minnesota, Minneapolis, MN 55455

The behavior of an electron probe inside a specimen has critical influence on the data collected from scattering events of the electron probe. This has been reported on several occasions in scanning transmission electron microscope (STEM) work, such as in detecting dopant atoms [1,2] and in measuring interatomic distances [3]. These observations necessitate a clear understanding of beam behavior inside a specimen for designing optimal STEM experiments and for correct interpretation of STEM data. A particular feature of the beam behavior that complicates quantitative analysis of ADF-STEM and EELS data is oscillation in beam intensity, known as channeling.

Electron probe channeling is observed not only during transmission through 3-d crystals, but also in the simpler case of transmission through one column of atoms [4]. Figure 1 compares probe profiles in $\mathrm{Si}$ crystal and in a hypothetical isolated column of $\mathrm{Si}$ atoms. Here we have characterized the electron beam intensity profile during transmission through an isolated column of atoms and describe the influence of neighboring columns.

Characteristics of the STEM probe intensity during transmission through a specimen are described via simulations based on Multislice method [5]. Effects of probe and specimen features on beam intensity profile are examined. Figure 2 shows beam intensity profiles for an ideal probe $\left(C_{s}=0\right)$ transmitted through isolated columns of group $\mathrm{V}$ elements. Figure 3 shows beam intensity profiles in isolated columns demonstrating that probe convergence angle causes little to no change in oscillation frequency, although it does affect intensity. Results shown in Figure 4 examine the effect of distance between atoms on channeling. It appears that different separation distances between atoms lead to similar oscillations in beam intensity.

Furthermore, we explain channeling with a physical picture based on Huygens principle. The wave front of the incident beam can be modeled as a discretized 2-d grid of spherical wavelets that interacts with discrete planes of atomic potential. The effect of elastic interaction between incident electron and atomic potential can be described as change in phase of the incident electron. Spherical wavelets at different locations in the x-y plane undergo different phase shifts, creating a phase gradient in the plane. The non-zero phase gradient leads to change in topology of the wave front. Repetitive change in phase gradient, resulting from repetitive interaction with planes of atomic potentials, leads to oscillation in beam intensity. We explored this picture in detail, elucidating fundamental reasons behind channeling.

\section{References}

[1] K. van Benthem et al., Ultramicroscopy, 106 (2006) 1062.

[2] A. Mittal and K. A. Mkhoyan, Ultramicroscopy 111 (2011) 1101.

[3] R. Hovden, H. Xin, and D. A. Muller, Phys. Rev. B 86 (2012) 195415.

[4] Z. Yu and J. Silcox, Microsc. Microanal. 10 (2004) 570. 
[5] E. J. Kirkland, “Advanced Computing in Electron Microscopy”. Springer (2010).

[6] This research was funded by Abu Dhabi Minnesota Institute for Research Excellence.
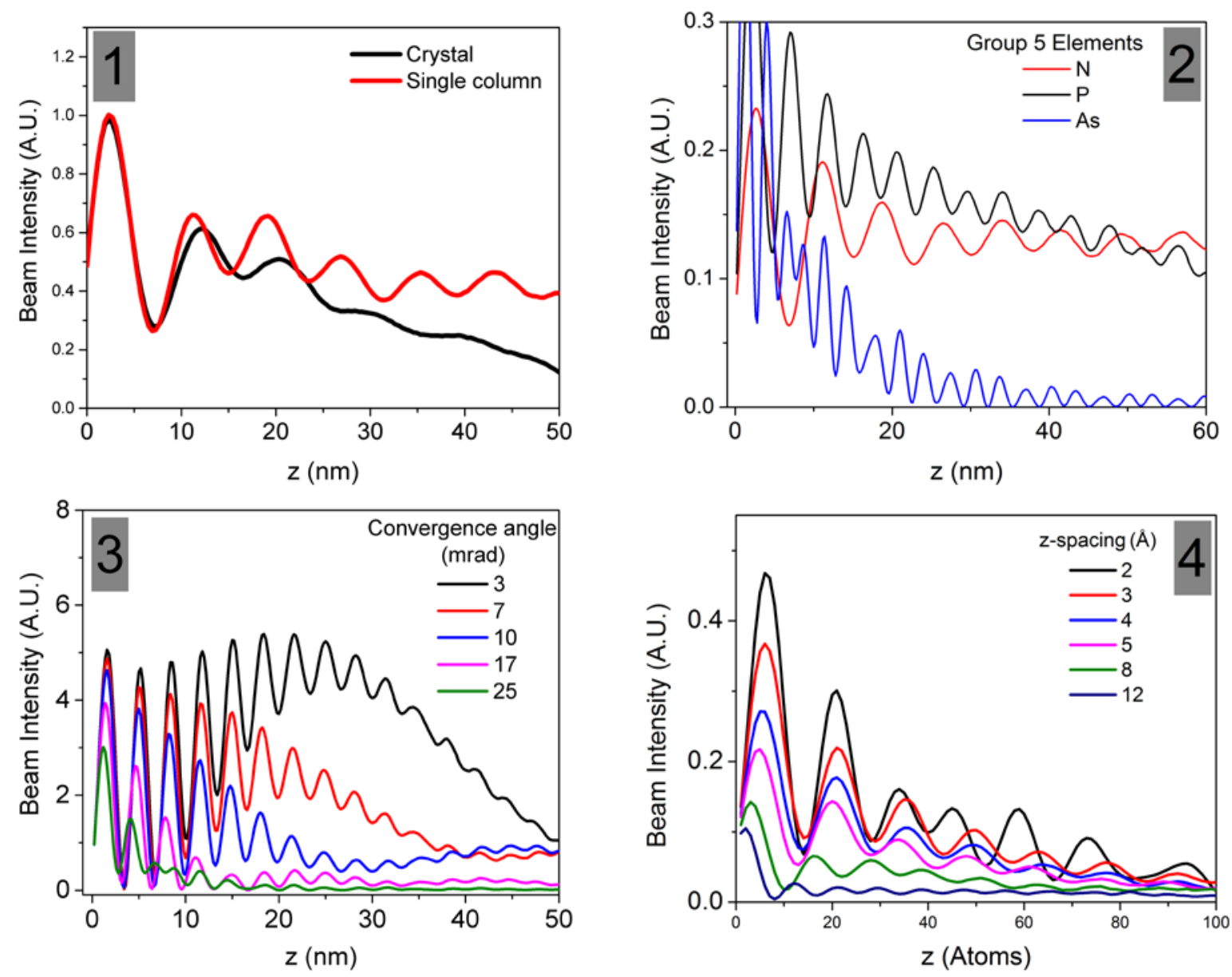

Fig. 1. Comparison of beam intensity profiles of $100 \mathrm{keV}, 25 \mathrm{mrad}$, ideal STEM probe in $\mathrm{Si}$ [110] and in an isolated atomic column of Si with the same atomic spacing as in Si [110].

Fig. 2. Beam intensity profiles of $100 \mathrm{keV}, 25 \mathrm{mrad}$, aberration-corrected STEM probe in isolated atomic columns of group V elements, with atoms spaced $2 \AA$ apart.

Fig. 3. Beam intensity profiles of $100 \mathrm{keV}$ ideal STEM probes with different convergence angles in isolated column of Ge with atoms spaced $2 \AA$ apart. Each profile scaled individually for display in one plot.

Fig. 4. Beam intensity profiles of $100 \mathrm{keV}, 25 \mathrm{mrad}$, aberration-corrected STEM probe in isolated Ge columns with different atomic spacings along the z-axis. 